\title{
Oftalmopatía de Graves eutiroidea con anticuerpos negativos
}

\section{Thyroid negative antibody euthyroid Graves' ophthalmopathy}

\author{
Obed A. Urquía-Sequeiros ${ }^{1 *}$, Alejandra Álvarez ${ }^{2}$ y Antonio Segovia-Palomo² \\ 'Servicio de Endocrinología; ${ }^{2}$ Clínica de Tiroides. Hospital General de México "Dr. Eduardo Liceaga", Ciudad de México, México
}

\section{RESUMEN}

La oftalmopatía de Graves es la principal afección extratiroidea en los pacientes con enfermedad de Graves. Es ocasionada, fundamentalmente, por la interacción de anticuerpos contra el receptor de tirotropina (AcTSH-R) con el receptor de tirotropina expresado en los fibrocitos orbitarios. Ocurre en un bajo porcentaje de pacientes eutiroideos. Se presenta el caso de paciente masculino de 50 años, sin antecedentes patológicos, con proptosis bilateral, CAS 6/7, EUGOGO moderado-severo; perfil y anticuerpos tiroideos normales. Se manejó con metilprednisolona y micofenolato. Posterior a la conclusión del tratamiento con esteroides presenta perfil tiroideo normal y AcTSH-R elevados.

Palabras clave: Oftalmopatía de Graves. Enfermedad de Graves. Anticuerpos contra el receptor de tirotropina. Eutiroidea.

\begin{abstract}
Graves 'ophthalmopathy is the main extra-thyroid condition in patients with Graves' disease. It is mainly caused by the interaction of antibodies against the thyrotropin receptor with the thyrotropin receptor expressed in orbital fibrocytes. It occurs in a low percentage of euthyroid patients, in these cases the measurement of antibodies is important for their diagnosis. Approaching patients with negative antibodies represents a diagnostic challenge. We present the case of a 50-year-old male patient, with no pathological history, with bilateral proptosis, CAS 6/7, moderate-severe EUGOGO; normal thyroid profile and antibodies. It was managed with boluses of methylprednisolone and mycophenolate. During follow-up, after completion of bolus treatment, control laboratories showed persistence of normal thyroid function, negative anti-peroxidase antibodies and elevated anti-receptor antibodies.
\end{abstract}

Key words: Grave's ophthalmopathy. Grave's disease. Thyrotropin receptor antibodies. Euthyroid.
Correspondencia:

*Obed A. Urquía-Sequeiros

E-mail: obedurquia@gmail.com
Fecha de recepción: 26-06-2020

Fecha de aceptación: 21-07-2020

DOI: 10.24875/RME.20000071
Disponible en internet: 02-06-2021 Rev Mex Endocrinol Metab Nutr. 2021;8:104-7

2462-4144 / ๑ 2020 Sociedad Mexicana de Nutricion y Endocrinologia, AC. Publicado por Permanyer. Éste es un artículo open access bajo la licencia CC BY-NC-ND (http://creativecommons.org/licenses/by-nc-nd/4.0/). 


\section{INTRODUCCIÓN}

La oftalmopatía de Graves (OG) es un trastorno inflamatorio de origen autoinmunitario que afecta la órbita y estructuras periorbitarias, y puede poner en riesgo la visión en algunos casos ${ }^{1}$. Representa la principal manifestación extratiroidea de la enfermedad de Graves (EG) ${ }^{2}$, encontrándose en un $20-25 \%$ de estos pacientes ${ }^{1}$. Es cinco veces más frecuente en mujeres, con un pico de incidencia en la $4 .^{a}$ a $6 .^{a}$ década de vida ${ }^{3}$. La OG usualmente se presenta de manera simultánea o poco después del inicio de la tirotoxicosis, rara vez precede al desarrollo de esta, menos del $10 \%$ de los pacientes son eutiroideos o hipotiroideos $^{4}$. Clínicamente se caracteriza por la presencia de retracción del párpado superior, edema periorbitario, proptosis y deterioro de la motilidad ocular, entre otros datos clínicos que han permitido la descripción de tres subtipos: miopática, congestiva y mixta ${ }^{2}$.

La fisiopatogenia está fundamentada en la interacción de anticuerpos antirreceptor de tirotropina (AcTSH-R) con el receptor de tirotropina (TSH) expresado en los fibrocitos orbitarios ${ }^{5}$. De tal forma que, para su diagnóstico, adicional a la evaluación de las características clínicas es necesario realizar determinación de pruebas de funcionamiento tiroideo, medición de AcTSH-R y estudios de imagen de las órbitas ${ }^{6}$. El perfil habitual suele ser el de un paciente con afección orbitaria y pruebas de funcionamiento tiroideo anormales con anticuerpos elevados. El reto diagnóstico surge cuando un paciente con oftalmopatía tiene niveles de anticuerpos y pruebas de función tiroidea normales.

\section{DESCRIPCIÓN DEL CASO}

Paciente de género masculino de 50 años, sin antecedentes de distiroidismo familiar; tabaquismo activo desde los 18 años (aproximadamente 10 cigarros/día). Refiere proptosis de ojo derecho desde hace nueve años y hace un año en ojo izquierdo, niega síntomas de disfunción tiroidea. Sin antecedente personal o familiar de enfermedad tiroidea.

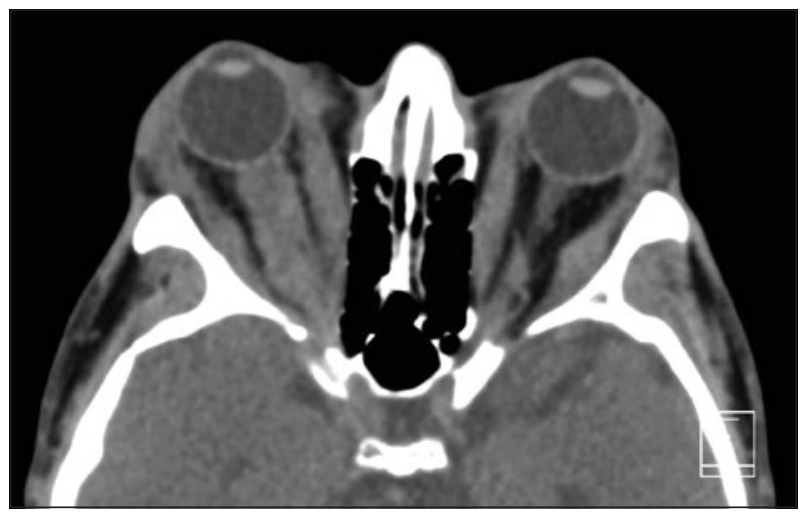

Figura 1. Tomografía axial computarizada de órbitas con engrosamiento de músculos extraoculares de predominio derecho.

Clínicamente eutiroideo sin bocio. Exoftalmometría: base, $110 \mathrm{~mm}$; ojo derecho (OD), $24 \mathrm{~mm}$; ojo izquierdo (OI), $23 \mathrm{~mm}$; Clinical Activity Socre (CAS) 6/7; ATA (Clasificación de severidad según la American Thyroid Association) clase III; clasificación EUGOGO, moderada-severa/activa. Pruebas de función tiroidea: TSH 3.02 (0.27-4.4 mUl/ml), triyodotironina libre (T3L) $3.6(2.0-4.4 \mathrm{pg} / \mathrm{ml})$, tiroxina libre (T4L) $0.93(0.93-1.70 \mathrm{ng} / \mathrm{dl})$, anticuerpos antiperoxidasa tiroidea (Ac anti-TPO) $<1$ (negativo $<5.6 \mathrm{Ul} / \mathrm{ml}$ ), AcTSH-R: $1.3 \mathrm{UI} / \mathrm{l}$ (negativo $<1.8$, indeterminado 1.8-2.0, positivo $>2.0$ ) cuantificado por inmunoensayo enzimático. Ultrasonido y Doppler tiroideo reportando glándula homogénea, de tamaño normal, sin nódulos, ecogenicidad discretamente disminuida de forma global, sin nódulos, patrón vascular tipo 2; pico sistólico de tiroideas inferiores: derecha $20.5 \mathrm{~cm} / \mathrm{s}$, izquierda $25.7 \mathrm{~cm} / \mathrm{s}$. Tomografía computarizada de órbitas con aumento bilateral del grosor de los músculos extraoculares (Fig. 1). Ante los resultados de pruebas de función e imagen tiroidea dentro de la normalidad, se presenta caso a Oftalmología, quienes realizan biopsia de músculo recto media derecho. El resultado histopatológico reporta: atrofia muscular leve/fibrosis (Fig. 2). Por lo anterior se consensuó tratamiento intravenoso con metilprednisolona y micofenolato de mofetilo $1 \mathrm{~g} / \mathrm{d}$, aunado a medidas generales de protección ocular y asesoría por clínica de tabaquismo. Durante el tratamiento con mejoría clínica, se completa esquema de metilprednisolona con una dosis total de $4.5 \mathrm{~g}$ en 12 semanas. Al finalizar el ciclo de glucocorticoide: exoftalmometría OD $21 \mathrm{~mm}$, OI $20 \mathrm{~mm}$, CAS 


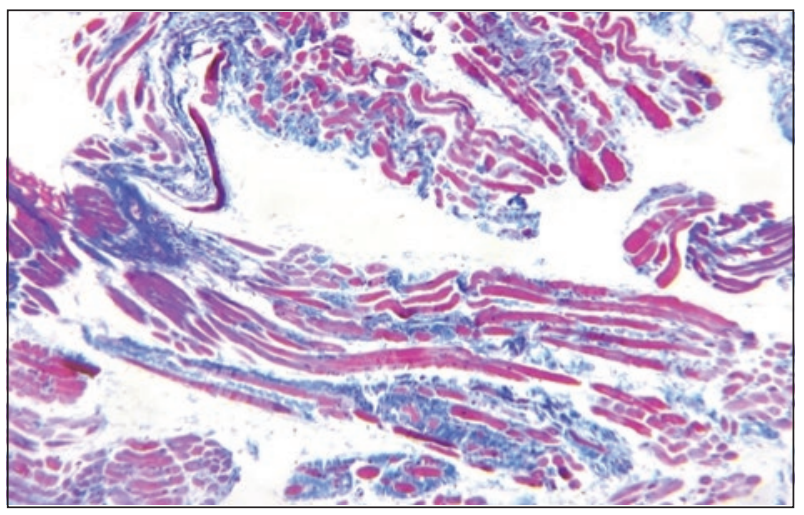

Figura 2. Tinción del músculo recto medial derecho con tricrómico de Masson, con evidente acumulación de las fibras de tejido conectivo de color azul (ampliación 10X).

3/10, ATA clase II, EUGOGO leve/inactiva. Pruebas de función hepática y biometría hemática normales, TSH $1.21(0.35-4.94 \mathrm{mUl} / \mathrm{ml})$, T3L 2.94 (1.88-3.18 pg/ $\mathrm{ml})$, T4L $0.94(0.7-1.48 \mathrm{ng} / \mathrm{dl})$, triyodotironina total $107.77(64-152 \mathrm{ng} / \mathrm{dl})$, tiroxina total $6.59(4.8-11.7$ $\mu \mathrm{g} / \mathrm{dl}$ ), AcTSH-R $3.8 \mathrm{UI} / \mathrm{l}$ (negativo < 1.0, indeterminado 1-1.5, positivo $>1.5$ ) cuantificado por ELISA, Ac TPO $<1$ (negativo $<5.6 \mathrm{Ul} / \mathrm{ml}$ ). Se decidió continuar tratamiento con micofenolato $500 \mathrm{mg} / \mathrm{d}$ por tres meses y suspensión de tabaquismo.

\section{DISCUSIÓN}

Los pacientes con EG y función tiroidea normal que desarrollan compromiso orbitario tienen oftalmopatía de Graves eutiroidea (OGE). El diagnóstico se demuestra por la presencia de AcTSH- $\mathrm{R}^{6}$. La prevalencia de AcTSH-R en OGE varía (0-100\%). Los pacientes con OGE tienen una enfermedad leve, unilateral o asimétrica y grados menores de inflamación de los tejidos blandos y afectación muscular ${ }^{7}$. Los niveles positivos de AcTSH-R, evento patogénico central en el inicio de OG, confirman el diagnóstico hasta en el $69 \%$ de los pacientes eutiroideos e hipotiroideos ${ }^{8}$.

El origen de la OG se centra en los fibroblastos de la órbita, ya que expresan receptores de TSH, siendo estos el blanco de anticuerpos, generando diferenciación de los adipocitos de la órbita, infiltración de linfocitos $\mathrm{T}$, con la consecuente producción de citocinas, además se activa la producción de colágeno y glucosaminoglucanos, siendo estos compuestos químicos hidrofílicos, produciendo expansión de los tejidos de la órbita secundario a la retención de agua ${ }^{9,10}$.

El receptor del factor de crecimiento 1 similar a la insulina, presente en la superficie de los fibroblastos orbitales, también se ha implicado en la patogénesis de la $\mathrm{EG}^{11}$.

Diversos estudios apoyan el rol de los anticuerpos dirigidos a otros antígenos del músculo conjuntivo y del tejido conectivo orbitario, tales como la proteína de unión al calcio calsecuestrina y el antígeno de membrana de fibroblastos orbitales colágeno XIII, los cuales están implicados en la inflamación de los músculos extraoculares, en especial los superioires $^{12,13}$, siendo esta una explicación alternativa para los pacientes con OG y AcTSH-R negativos.

La OGE con función tiroidea normal y anticuerpos tiroideos negativos es poco común ${ }^{14}$. La causa de la negatividad de AcTSH-R no está clara, se ha sugerido la existencia de AcTSH-R intratiroideos. Los niveles de AcTSH-R son muy bajos en pacientes con OGE y disminuyen con el tiempo, correlacionando con fenotipos de enfermedades más leves en pacientes con AcTSH-R negativo ${ }^{15}$.

El tratamiento de elección para la OGE y AcTSH-R negativos es la terapia con corticosteroides sistémicos ${ }^{16}$. En nuestro paciente se utilizó un esquema de bolos de metilprednisolona y micofenolato, con lo cual se logró mejoría tanto en datos clínicos de actividad como de severidad. Esquema terapéutico ya evaluado en el estudio MINGO, donde la adición de micofenolato a metilprednisolona, si bien no demostró cambios en la tasa de recaída a las 24 y 36 semanas, evidenció un incremento en la tasa de respuesta (71 vs. 53\%; odds ratio: 2.16: intervalo de confianza: 1.09-4.25; $\mathrm{p}=0.026)$ a las 24 semanas de tratamiento en pacientes con oftalmopatía moderada-severa activa ${ }^{17}$.

El paciente se ha mantenido eutiroideo durante el seguimiento. A pesar del incremento en los niveles de AcTSH-R, esta elevación probablemente se deba $a$ isoformas neutras $y / o$ bloqueantes o activación conjunta de la vía de diacilglicerol y adenosina monofosfato cíclico ${ }^{18}$. Entre el 4 y el $18 \%$ de los pacientes con EG desarrollan disfunción tiroidea después 
del inicio de oftalmopatía, la mayoría dentro de un año. El $25 \%$ de los pacientes principalmente eutiroideos desarrollarán disfunción tiroidea en cuatro $a_{\text {ños }}^{4,6}$. Los predictores de esto son TSH suprimida y AcTSH-R positivos ${ }^{15}$.

\section{CONCLUSIÓN}

Se presenta el caso de un paciente con OGE con anticuerpos negativos, de manera inicial; una forma de presentación infrecuente, que por ende representa un reto diagnóstico y terapéutico, siendo necesario el abordaje multidisciplinario. La respuesta al plan terapéutico puede contribuir a mantener el régimen establecido y al diagnóstico final, como ocurrió en este paciente.

\section{FINANCIAMIENTO}

Los autores no recibieron patrocinio para llevar a cabo este artículo.

\section{CONFLICTO DE INTERESES}

Los autores declaran no tener conflicto de intereses alguno.

\section{RESPONSABILIDADES ÉTICAS}

Protección de personas y animales. Los autores declaran que para esta investigación no se han realizado experimentos en seres humanos ni en animales.

Confidencialidad de los datos. Los autores declaran que han seguido los protocolos de su centro de trabajo sobre la publicación de datos de pacientes.
Derecho a la privacidad y consentimiento informado. Los autores han obtenido el consentimiento informado de los pacientes y/o sujetos referidos en el artículo. Este documento obra en poder del autor de correspondencia.

1. Barrio-Barrio J, Sabater AL, Bonet-Farriol E, Velázquez-Villoria Á, Galofré JC. Graves' Ophthalmopathy: VISA versus EUGOGO classification, assessment, and management. J Ophthalmol. 2015;2015:249125.

2. Luster M, Duntas L, Wartofsky L. The thyroid and its diseases. 1st ed. Suiza: Springer International Publishing: 2019. pp. 323-325.

3. Bartalena L, Baldeschi L, Boboridis K, Eckstein A, Kahaly GJ, Marcocci C, et al. The 2016 European Thyroid Association/European Group on Graves' Orbitopathy Guidelines for the Management of Graves' Orbitopathy. Eur Thyroid J. 2016;5:9-26.

4. Eckstein AK, Lösch C, Glowacka D, Schott M, Mann K, Esser J, et al. Euthyroid and primarily hypothyroid patients develop milder and significantly more asymmetrical Graves ophthalmopathy. Br J Ophthalmol. 2009;93(8):1052-6.

5. Bahn RS. Graves' ophthalmopathy. N Engl J Med . 2010;362(8):726-38.

6. Tabasum A, Khan I, Taylor P, Das G, Okosieme OE. Thyroid antibody-negative euthyroid Graves' ophthalmopathy. Endocrinol Diabetes Metab Case Rep. 2016;2016:160008.

7. Strianese D, Piscopo R, Elefante A, Napoli M, Comune C, Baronissi I, et al. Unilateral proptosis in thyroid eye disease with subsequent contralateral involvement: retrospective follow-up study. BMC Ophthalmol. 2013;13:21.

8. Suzuki N, Noh JY, Kameda T, Yoshihara A, Ohye H, Suzuki M, et al. Clinical course of thyroid function and thyroid associated-ophthalmopathy in patients with euthyroid Graves' disease. Clin Ophthalmol. 2018;12:739-46.

9. Mallika P, Tan A, Aziz S, Alwi SS, Chong Ms, Vanitha R, et al. Thyroid associated ophthalmopathy - a review. Malays Fam Physician. 2009;4(1):8-14.

10. Braverman LE, Cooper D. Werner \& Ingbar's The thyroid: A fundamental and clinical text. 10th ed. Filadelfia: Lippincott Williams \& Wilkins; 2012. pp. 369-370.

11. Smith TJ, Janssen JA. Insulin-like growth factor-I receptor and thyroidassociated ophthalmopathy. Endocrine Rev. 2019;40:236-67.

12. Lahooti $\mathrm{H}$, Parmar KR, Wall JR. Pathogenesis of thyroid-associated ophthalmopathy: does autoimmunity against calsequestrin and collagen XIII play a role? Clin Ophthalmol. 2010;4:417-25.

13. Kan E, Kan EK, Ecemis G, Colak R. Presence of thyroid-associated ophthalmopathy in Hashimoto's thyroiditis. Int J Ophthalmol. 2014;7(4):644-7.

14. Yu P, Liu S, Zhou X, Huang T, Li Y, Wang H, et al. Thyroid-associated orbitopathy in patients with thyroid carcinoma: A case report of 5 cases. Medicine (Baltimore). 2017;96(47):e8768.

15. Khoo DH, Eng PH, Ho SC, Tai ES, Morgenthaler NG, Seah LL, et al. Graves' ophthalmopathy in the absence of elevated free thyroxine and triiodothyronine levels: prevalence, natural history, and thyrotropin receptor antibody levels. Thyroid. 2000;10:1093-100.

16. Zang S, Ponto KA, Kahaly GJ. Clinical review: Intravenous glucocorticoids for Graves' orbitopathy: efficacy and morbidity. J Clin Endocrinol Metab. 2011;96(2):320-32

17. Kahaly GJ, Riedl M, König J, Pitz S, Ponto K, Diana T, et al. Mycophenolate plus methylprednisolone versus methylprednisolone alone in active, moderate-to-severe Graves' orbitopathy (MINGO): a randomised, observer-masked, multicentre trial. Lancet Diabetes Endocrinol. 2018;6(4):287-98.

18. Latif $R$, Morshed SA, Zaidi M, Davies TF. The thyroid-stimulating hormone receptor: impact of thyroid-stimulating hormone and thyroid-stimulating hormone receptor antibodies on multimerization, cleavage, and signaling. Endocrinol Metab Clin North Am. 2009;38:319-41. 